\title{
Characteristics of Indigenous adults with poorly controlled diabetes in north Queensland: implications for services
}

David Ross Johnson ${ }^{1 *}$, Robyn Anne McDermott ${ }^{2}$, Peter Marshall Clifton ${ }^{3}$, Katina D’Onise ${ }^{4}$, Sean Matthew Taylor ${ }^{2}$, Cilla Louise Preece ${ }^{4}$ and Barbara Anne Schmidt ${ }^{2,4}$

\begin{abstract}
Background: Indigenous Australian adults with diabetes continue to have suboptimal clinical control and poorer outcomes compared with non-Indigenous people although there is a paucity of data documenting the detailed health status of Indigenous people in Australia. To further investigate the characteristics of Indigenous Australian adults with poorly controlled diabetes we analysed baseline data from a cluster randomized trial aiming to deliver a program of integrated community-based intensive chronic disease management for Indigenous people in remote communities in far north Queensland, Australia.
\end{abstract}

Methods: Indigenous adults aged 18 to 65 years from 12 clinics in rural north Queensland with established type 2 diabetes and with $\mathrm{HbA} 1 \mathrm{c} \geq 8.5 \%$ were invited to participate. The primary outcome variable measured at baseline was $\mathrm{HbA1c}$. Other variables measured included socio-demographic indicators, health literacy, BMl, blood pressure, lipids, renal function, smoking status and quality of life measures. Data were collected between December 2010 and July 2011. Analysis was performed by ethnicity - Aboriginal or Torres Strait Islander.

Results: One hundred and ninety three participants were included in the analysis. Very high rates of albuminuria, high rates of smoking, dyslipidaemia, hypertension and elevated BMI were recorded. Aboriginal participants reported higher levels of socio-economic disadvantage, higher smoking rates, lower BMI and worse self-reported health status than Torres Strait Islander participants.

Conclusion: These results demonstrate a high potential for improved culturally sound community-based management of diabetes and other comorbid conditions in this very high risk population. They also provide further evidence for including albuminuria in cardiovascular risk calculation.

Keywords: Diabetes mellitus type 2, Indigenous, Albuminuria, Risk factors, Primary health care

\section{Background}

Indigenous Australians experience very high prevalence, morbidity and mortality from chronic health conditions such as diabetes, cardiovascular, renal and chronic respiratory disease [1-3]. The higher rates of these conditions accounts for the majority of the 'health gap' between Indigenous and non-Indigenous Australian's with those residing in remote areas particularly affected [4]. As a result, Indigenous people experience much

\footnotetext{
* Correspondence: david.johnson@ahcsa.org.au

${ }^{1}$ Aboriginal Health Council of South Australia, 9 King William Road, Adelaide, SA 5061, Australia

Full list of author information is available at the end of the article
}

higher rates of avoidable hospital admissions from chronic diseases [5,6] and, while the causes of this are complex, a lack of access to effective primary health care services is a major contributing factor [7].

Over recent years there has been an increasing focus both internationally [8] and in Australia $[9,10]$ on enhancing the capacity of primary care delivery systems, originally set up to manage acute disease, to better prevent and manage chronic diseases and their complications. The Chronic Care Model has been influential in enhancing chronic illness care through improving clinical system delivery design, clinical decision support, clinical information systems, patient self-management 
support, organizational support and mobilizing community resources to better manage chronic disease [8].

At the same time, a number of Indigenous community health services in Australia have undertaken chronic disease quality improvement initiatives adopting many of the Chronic Care model components [11]. While improvements have been made in the provision of routine clinical care processes, there has been less success translating this into improved intermediate clinical outcomes, such as glycaemic control for people with diabetes or reduction in risk factors such as overweight and obesity $[12,13]$. This has been in part attributed to what has been termed the 'clinical inertia' of treating doctors [14], particularly relating to delays in initiating insulin [15]. More importantly, perhaps, has been the under-utilisation of the Indigenous Health workforce. In Australia, there is good evidence that Indigenous Health Workers (IHWs) are well placed to provide routine chronic disease care via the use of disease management protocols which is cost effective as long as there is: (1) adequate training in chronic disease management and health promotion/disease prevention; (2) good clinical support mechanisms and; (3) system-level support to ensure adequate staffing to enable chronic disease selfmanagement support for individuals, families and groups in community settings [16-18].

Recognising the central role played by IHWs, the "Getting Better at Chronic Care" (GBACC) study in North Queensland is a randomized cluster trial that aims to deliver integrated community-based, intensive chronic disease management initially in a small number of Indigenous communities in the Torres Strait, Cape York and Cairns districts in far North Queensland, Australia.

This paper reports on selected baseline clinical and socioeconomic data from this study.

\section{Methods}

Study design, setting and participant selection

This is a baseline analysis of the GBACC study whose methodology has been described elsewhere [19]. In summary, this is an open parallel randomised cluster trial delivering integrated community-based, intensive chronic disease management, compared to usual care, via rural and remote Indigenous primary health care services to Indigenous Australians living in far North Queensland, Australia. Twelve clinics (clusters) in rural north Queensland were chosen based on having significant Indigenous populations and primary health care services provided either by Queensland Health or a local communitycontrolled Indigenous Health Service. Potential participants were identified from diabetic disease registers of the participating health services. Adults aged 18 to 65 years from these registers with established type 2 diabetes (diagnosed for more than one year) and with HbA1c $\geq 8.5 \%$ were invited to participate in the study. Written informed consent was obtained from individual participants. Data collection was undertaken between December 2010 and July 2011.

\section{Variables}

The primary outcome variable measured at baseline was HbA1c. Other variables measured included sociodemographic indicators, health literacy, intermediate clinical outcomes other than HbA1c (renal function, blood pressure, lipids), preventative and patient selfcare practices and quality of life measures.

Ethnicity was based on self-report. Where participants reported "both Torres Strait Islander and Aboriginal" descent they were classified as Torres Strait Islander for this analysis. Health literacy was measured using the Test Of Functional Health Literacy in Adults (TOFHLA) tool (score 0-100; 0-59 inadequate, 60-74 marginal, 75-100 adequate health literacy) [20]. Renal function was calculated from serum creatinine using estimated Glomerular Filtration Rate (eGFR) calculated by the Chronic Kidney Disease Epidemiology Collaboration (CKD-EPI) formula [21] and albuminuria was defined as a urinary albumin creatinine ratio (ACR) $\geq 2.5 \mathrm{mg} / \mathrm{mmol}$ in men and $\geq 3.5 \mathrm{mg} / \mathrm{mmol}$ in women. Chronic kidney disease (CKD) stage was calculated as per the Kidney Health Australia Caring for Australasians with Renal Impairment (KHA-CARI) guidelines [22]. Those with CKD stage 4 and 5 were excluded from the study. Hypertension was defined as blood pressure $>140 / 90$ or if prescribed an antihypertensive medication. Dyslipidaemia was defined as one or more of total cholesterol (TC) $\geq 4 \mathrm{mmol} / \mathrm{L}$, highdensity lipoprotein cholesterol (HDL-C) $<1 \mathrm{mmol} / \mathrm{L}$ and triglyceride (TG) $\geq 1.5 \mathrm{mmol} / \mathrm{L}$. Quality of life was measured using the Assessment of Quality of Life (AQoL) instrument and the 36-Item Short-Form (SF-36v2) health survey. The TOFHLA tool, AQoL and SF-36v2 surveys as well as a personal information survey were administered face-to-face with participants. All clinical data was obtained via review of hard copy primary health care charts, patient information management systems (Ferret/Medical Director/Best Practice as applicable) and Auslab/Auscare databases for pathology.

\section{Ethics approval}

The GBACC study was approved by the University of South Australia Human Research Ethics Committee, the University of Queensland Medical Research Ethics Committee and the Cairns and Hinterland Health Service District Human Research Ethics Committee with support from the relevant peak Aboriginal and Torres Strait Islander Health Councils (Trial Registration number 12610000812099). This project has a high level of community engagement through partnering with the local 
regional Indigenous health organisation, local Indigenous health services and community councils of participating communities. The potential benefits of the study are large both at the individual and family level as well as for the Indigenous health workforce. Further, a major strength of the project is its focus on sustainability which will be achieved by building capability of the local Indigenous health workforce.

\section{Statistical analyses}

Data were analysed by ethnicity as Torres Strait Islanders are genetically, culturally and geographically distinct from mainland Aboriginal Australians. We were interested in exploring clinical as well as socio-demographic differences between these populations which may have relevance to diabetes/chronic disease management. Chi square tests were used to compare proportions, student's t-tests for comparison of categorical and normally distributed continuous variables, Kruskal-Wallis tests for comparison of categorical and non-normally distributed continuous variables and Spearman Rank correlation tests for comparison of non-normally distributed continuous variables. Linear regression was also performed to further assess associations. All analyses were undertaken using Stata version 11 (Stata Corp, TX, USA).

\section{Results}

One hundred and ninety three participants who met the modified inclusion criteria were included in the baseline data analysis. This represented approximately $75 \%$ of the eligible population on registers who were invited to participate. Equal numbers of Torres Strait Islander $(n=97)$ and Aboriginal people $(n=96)$ were represented.

\section{Socio-demographic characteristics for Aboriginal and Torres Strait Islander participants}

The mean age for participants was 48 years. Overall there were a higher proportion of female participants (64\%) and this was most marked for Aboriginal people (70\%) (Table 1).

A high number of participants were not currently employed (47\%) although it was lower for Torres Strait Islanders $(35 \%)$ than for Aboriginal people $(59 \%)(\mathrm{p}=$ 0.003). Aboriginal participants were also less likely to have completed year $12(\mathrm{p}=<0.001)$, reported lower median incomes $(p=<0.001)$, were more likely to not have enough money for food at times $(\mathrm{p}<0.001)$ and were more likely to have missed meals in the previous fortnight $(\mathrm{p}<0.001)$ compared to Torres Strait Islanders (Table 1).

Despite this, there was little difference in median TO FHLA scores by ethnicity but they were higher for those who had completed year $12(8.3,95 \% \mathrm{CI}, 2.6$ to $13.9, \mathrm{p}=$ $0.004)$ after controlling for age, sex and ethnicity.
Overall the proportion of smokers was high (41\%) with Aboriginal people more likely to smoke (46\%) compared to Torres Strait Islanders (35\%) (Table 1). Most of the difference in smoking prevalence was accounted for by a higher proportion of female Aboriginal smokers. Torres Strait Islander mean body mass index (BMI) scores were much higher than for Aboriginal people (BMI score 4.3, 95\% CI, 1.8 to $6.7, \mathrm{p}=0.001$ ) after controlling for smoking, age and sex.

\section{Intermediate clinical outcomes and preventive care practices}

Even though only participants with $\mathrm{HbA} 1 \mathrm{c} \geq 8.5 \%$ were included, median $\mathrm{HbA1c}$ overall was still very high (10.9\%) (Table 2). There was a weak association observed between lower HbA1c and higher TOFHLA score although this was unlikely to be clinically important (for a 10 point increase in TOFHLA score HbA1c decreased by $0.23 \%, 95 \% \mathrm{CI},-0.49$ to $+0.029 \%, \mathrm{p}=0.082$ ).

A high proportion were diagnosed with hypertension and, although overall mean systolic and diastolic readings suggested reasonable blood pressure control, nearly half of those with a diagnosis of hypertension recorded blood pressure readings $>140 / 90$.

The proportion of those with albuminuria was extremely high (75\%) and the majority of participants were classified as having stage 1 to stage 3 chronic kidney disease (73\%) with CKD stage being slightly less advanced for Aboriginal compared to Torres Strait Islander participants. Overall, mean high-density lipoprotein cholesterol (HDL-C) was low $(0.98 \mathrm{mmol} / \mathrm{L})$ with only $32 \%$ of participants recording normal HDL levels. Mean total cholesterol: HDL-C ratios were also high (5.3).

Forty per cent of participants were prescribed insulin and $43 \%$ monitored their own blood sugars.

The majority of those with a diagnosis of hypertension were prescribed an anti-hypertensive medication (92\%) and relatively high proportions of those with both albuminuria and dyslipidaemia were presribed reninangiotensin inhibitors (angiotensin converting enzyme inhibitors and/or angiotensin receptor blockers) (85\%) and lipid lowering medication (75\%) respectively. Torres Strait Islander participants were less likely to be adherent to all medications $(\mathrm{p}=0.02)$ (Table 2). Insulin use was not associated with medication adherence.

\section{Selected quality of life and self-reported health measures}

Although Aboriginal participants were less likely to be anxious or depressed, they reported more pain and tiredness and they were less likely to report excellent or very good health compared to Torres Strait Islanders (Table 3). 
Table 1 Socio-demographic indicators by ethnicity, chronic care study, 2011 ( $N=193+)$

\begin{tabular}{|c|c|c|c|c|}
\hline & Aboriginal & Torres Strait Islander & Overall & P-value \\
\hline Number of participants & 96 & 97 & 193 & \\
\hline Mean (95\% Cl) age (years) & $47.7(45.9-49.6)$ & $47.5(45.5-49.5)$ & $47.6(46.2-49.0)$ & 0.92 \\
\hline Number (\%) of women & $67(70)$ & $56(58)$ & $123(64)$ & 0.08 \\
\hline Employment status (\%) & & & & 0.003 \\
\hline Full-time & $25(26)$ & $41(42)$ & $66(34)$ & \\
\hline Part-time & $14(15)$ & $22(23)$ & $36(19)$ & \\
\hline Unemployed & $57(59)$ & $34(35)$ & $91(47)$ & \\
\hline Education level attained (\%) & & & & $<0.001$ \\
\hline Did not complete year 12 & $77(80)$ & $52(54)$ & $129(67)$ & \\
\hline Year 12 certificate & $2(2)$ & $16(16)$ & $18(9)$ & \\
\hline TAFE course & $14(15)$ & $19(20)$ & $33(17)$ & \\
\hline University course & $3(3)$ & $10(10)$ & $13(7)$ & \\
\hline Median (interquartile range) income (\$) & $15080(11960-27690)$ & $28600(14300-36400)$ & $18980(13000-33800)$ & $<0.001$ \\
\hline Not enough money for food (\%) & $49(51)$ & $25(26)$ & $74(38)$ & $<0.001$ \\
\hline No. of missed meals past fortnight (\%) & & & & $<0.001$ \\
\hline None & $49(55)$ & $93(98)$ & $142(77)$ & \\
\hline One to two & $26(29)$ & $1(1)$ & $27(15)$ & \\
\hline Three or more & $14(16)$ & $1(1)$ & $15(8)$ & \\
\hline Median (interquartile range) TOFHLA score & $86.2(72.5-94.0)$ & $86.2(68.8-91.1)$ & $86.2(71.1-93.0)$ & 0.23 \\
\hline No. of people per house (interquartile range) & $5(3-7)$ & $4(3-7)$ & $4(3-7)$ & 0.84 \\
\hline \multicolumn{5}{|l|}{ Smoking } \\
\hline Current smoking (\%) & $43(46)$ & $33(35)$ & $76(41)$ & $0.13 \neq$ \\
\hline Never smoked (\%) & $33(35)$ & $37(40)$ & $70(38)$ & \\
\hline Ex-smoker (\%) & $17(18)$ & $23(25)$ & $40(21)$ & \\
\hline Current female smoker (\%) & $33(50)$ & $20(36)$ & $53(44)$ & \\
\hline Current male smoker (\%) & $10(37)$ & $13(34)$ & $23(35)$ & \\
\hline Mean $(95 \% \mathrm{Cl})$ body mass index $(\mathrm{kg} / \mathrm{m} 2)$ & $30.3(28.6-32.0)$ & $34.2(32.2-36.2)$ & $31.9(30.6-33.3)$ & 0.004 \\
\hline
\end{tabular}

†Missing data: Missed meals 9, Smoking 7, BMI 106.

$¥$ Smoker/non-smoker as binary.

\section{Discussion}

This Indigenous population with poorly controlled diabetes recorded very high rates of albuminuria, a nontraditional cardiovascular risk factor, as well as high rates of smoking, elevated BMI, dyslipidaemia and hypertension. Differences related to ethnicity included higher levels of socio-economic disadvantage, higher smoking rates, lower BMI and worse self-reported health status for Aboriginal people compared to Torres Strait Islander people.

The prevalence of albuminuria (77\%) was very high for this group of Indigenous people. High rates of albuminuria have previously been reported in Indigenous populations in Australia with and without diabetes [23-26] most pronounced in remote locations [27] and at much higher levels than for non-Indigenous Australians [28,29]. In our study, despite the higher prevalence of albuminuria, median eGFR was still relatively high although our results are limited by the exclusion of those with CKD stage 4 and 5 .
Disproportionately high rates of albuminuria compared to eGFR have previously been reported in Indigenous populations [26,29] which may reflect different age profiles and rates of diabetic or obesity related hyper-filtration compared to non-Indigenous populations. Albuminuria may also be a better indicator of progression of CKD than eGFR in those with type 2 diabetes or it may reflect differences in the origins and pathophysiology of CKD in Indigenous versus non-Indigenous populations [26,30]. For our study eGFR was calculated using the CKD-EPI formula which provides better estimates compared to the MDRD formula [31].

There is good evidence that albuminuria independently predicts renal and cardiovascular risk in diabetics $[32,33]$ which is the leading cause of death for diabetics. Albuminuria can be reduced through the use of reninangiotensin inhibitors $[32,34]$. In this Indigenous population $85 \%$ of those with albuminuria were prescribed a 
Table 2 Baseline intermediate clinical outcomes, chronic care study, $2011(\mathrm{~N}=193+)$

\begin{tabular}{|c|c|c|c|c|}
\hline & Aboriginal & Torres Strait Islander & Overall & P-value \\
\hline Median (interquartile range) $\mathrm{HbA} 1 \mathrm{c}$ & $10.9(9.6-12.1)$ & $11.0(9.7-12.4)$ & $10.9(9.7-12.2)$ & 0.38 \\
\hline Hypertension diagnosed (\%) & $75(78)$ & $71(74)$ & $146(76)$ & 0.50 \\
\hline Mean $(95 \% \mathrm{Cl})$ systolic BP $(\mathrm{mmHg})$ & $131.0(126.9-135.2)$ & $131.6(127.6-135.7)$ & $131.3(128.5-134.2)$ & 0.84 \\
\hline Mean $(95 \% \mathrm{Cl})$ diastolic BP (mmHg) & $80.0(77.8-82.3)$ & $78.8(76.5-81.1)$ & $79.4(77.8-81.0)$ & 0.44 \\
\hline Hypertension diagnosed and normal BP $\ddagger$ (\%) & $38(51)$ & $39(55)$ & $77(53)$ & 0.61 \\
\hline Albuminuria (\%) & $56(76)$ & $47(73)$ & $103(75)$ & 0.76 \\
\hline $\begin{array}{l}\text { Median (interquartile range) estimated glomerular } \\
\text { filtration rate }\left(\mathrm{ml} / \mathrm{min} / 1.73 \mathrm{~m}^{2}\right)\end{array}$ & $103.9(88.6-111.4)$ & $94.7(77.1-115.7)$ & $100.6(80.9-113.0)$ & 0.27 \\
\hline Chronic kidney disease stage & & & & 0.08 \\
\hline Normal renal function & $17(24)$ & $19(29)$ & $36(27)$ & \\
\hline Stage 1 (\%) & $35(49)$ & $21(32)$ & $56(41)$ & \\
\hline Stage 2 (\%) & $15(21)$ & $14(22)$ & $29(21)$ & \\
\hline Stage $3(\%)$ & $4(6)$ & $11(17)$ & $15(11)$ & \\
\hline Total & 71 & 65 & 136 & \\
\hline Mean $(95 \% \mathrm{Cl})$ total cholesterol $(\mathrm{mmol} / \mathrm{L})$ & $4.7(4.4-5.0)$ & $4.6(4.3-4.9)$ & $4.6(4.4-4.8)$ & 0.53 \\
\hline Total cholesterol <4 mmol/L (\%) & $25(31)$ & $27(38)$ & $52(34)$ & 0.38 \\
\hline Mean $(95 \% \mathrm{Cl})$ high-density lipoprotein cholesterol (mmol/L) & $0.90(0.85-0.95)$ & $1.05(0.77-1.3)$ & $0.98(0.83-1.12)$ & 0.32 \\
\hline High-density lipoprotein cholesterol $\geq 1$ mmol/L (\%) & $25(38)$ & $18(26)$ & $43(32)$ & 0.13 \\
\hline Mean total cholesterol: HDL ratio $(95 \% \mathrm{Cl})$ & $5.3(4.9-5.7)$ & $5.3(4.9-5.7)$ & $5.3(5.0-5.6)$ & 0.88 \\
\hline Median (interquartile range) triglyceride ( $\mathrm{mmol} / \mathrm{L})$ & $2.2(1.5-3.5)$ & $1.7(1.1-2.8)$ & $2.0(1.2-3.1)$ & 0.02 \\
\hline Triglyceride <1.5 mmol/L (\%) & $19(24)$ & $33(46)$ & $52(35)$ & 0.004 \\
\hline Dyslipidaemia (\%) & $78(98)$ & $66(93)$ & $144(95)$ & 0.19 \\
\hline Taking insulin (\%) & $35(36)$ & $43(44)$ & $78(40)$ & 0.27 \\
\hline Monitors own blood sugar (\%) & $45(47)$ & $37(39)$ & $82(43)$ & 0.22 \\
\hline Adherent to all medications (\%) & $46(48)$ & $31(32)$ & $77(40)$ & 0.02 \\
\hline Prescribed anti-hypertensive medication if diagnosis of hypertension (\%) & $67(89)$ & $67(94)$ & $134(92)$ & 0.27 \\
\hline Albuminuria and prescribed renin-angiotensin inhibitors (\%) & $45(80)$ & $42(89)$ & $87(85)$ & 0.13 \\
\hline Prescribed lipid lowering medication (\%) & $77(80)$ & $68(70)$ & $145(75)$ & 0.10 \\
\hline
\end{tabular}

†Missing data: Systolic and Diastolic BP 9, Hypertension diagnosed 1, Albuminuria 55, CKD 54 (3 CKD stage 4 not included), TC 42, HDL-C 59, TG 43, Dyslipidaemia 42 , monitors blood sugar 2, sometimes miss medication 9 .

‡Defined as $<140 / 90$.

renin-angiotensin inhibitor. This is higher than the rate of renin-angiotensin blockade in an urban sample of both Indigenous Australians with diabetes (47\%) and non-Indigenous Australians with diabetes (66\%) [29]. However, it may be that despite the appropriate drugs being prescribed, the dose titration may not be adequate to impact most effectively for renal protection.

Only $40 \%$ of Indigenous participants were prescribed insulin despite the high glycaemic levels. This may still reflect a degree of clinical inertia [35] on the part of medical staff working with Indigenous clients, as they are not being treated as intensively as clinically indicated.

Although previous studies have shown inadequate health literacy is associated with worse glycaemic control [36,37], there was little evidence for such a relationship for Indigenous participants in our study. It may be that there was not the diversity of HbA1c measures to show such a relationship given that only people with uncontrolled diabetes were included. It was also interesting to note that despite the relative socioeconomic disadvantage, including lower year 12 completion rates, Aboriginal people had similar health literacy scores to Torres Strait Islanders. This suggests either limitations with the TOFHLA tool used in this setting or that other unmeasured factors were compensating for this disadvantage.

Women were over-represented in the Indigenous sample of people with uncontrolled diabetes. This has previously been shown in both remote and urban Indigenous Australian populations [38] as a result of higher rates of obesity in Indigenous women placing them at higher risk of diabetes [39]. This is in contrast to non-Indigenous populations where women and men are either equally 
Table 3 Quality of life measures by ethnicity, chronic care study, 2011 ( $N=193$ )

\begin{tabular}{|c|c|c|c|c|}
\hline & Aboriginal & Torres Strait Islander & Overall & P-value \\
\hline Anxious or depressed & & & & $<0.001$ \\
\hline No & $37(39)$ & $18(19)$ & $55(29)$ & \\
\hline Slightly & $47(49)$ & $75(77)$ & $122(63)$ & \\
\hline Moderately & $12(12)$ & $4(4)$ & $16(8)$ & \\
\hline Pain & & & & 0.01 \\
\hline None & $58(60)$ & $79(81)$ & $137(71)$ & \\
\hline Moderate & $31(32)$ & $15(16)$ & $46(24)$ & \\
\hline Severe & $5(5)$ & $3(3)$ & $8(4)$ & \\
\hline Unbearable & $2(2)$ & 0 & $2(1)$ & \\
\hline Self-reported health status & & & & 0.008 \\
\hline Excellent or very good & $12(13)$ & $30(31)$ & $42(22)$ & \\
\hline Good & $50(52)$ & $41(42)$ & $91(47)$ & \\
\hline Fair or poor & $34(35)$ & $26(27)$ & $60(31)$ & \\
\hline Tired & & & & $<0.001$ \\
\hline All or most of the time & $20(21)$ & $6(6)$ & $26(13)$ & \\
\hline Some of the time & $50(52)$ & $38(39)$ & $88(46)$ & \\
\hline A little or none of the time & $26(27)$ & $53(55)$ & 79 (41) & \\
\hline
\end{tabular}

Anxious or depressed Q14 from the Assessment of Quality of Life Survey (AQoL); pain Q15 from AQoL survey; self-reported health status Q1 from SF-36v2 Health Survey; tired Q9i from SF-36v2.

represented [29,38] or men over-represented [40]. In our sample, BMI was significantly lower for Aboriginal compared to Torres Strait Islander participants and this persisted after controlling for age, smoking status and sex. Such differences have previously been reported [28] and may be explained by a greater relative lean body mass for given height for Torres Strait Islanders given their genetically Melanesian origins [41,42]. Relative food insecurity as a result of relative socio-economic disadvantage for Aboriginal participants may also explain some of this difference in our study.

The mean age of people in our sample was very young. It has previously been shown that Indigenous Australians are diagnosed with diabetes at a much younger age than non-Indigenous Australians [28,29]. Possible explanations include a survival bias whereby Indigenous populations experience more risk factors (such as obesity and smoking) for diabetes and diabetes related complications resulting in higher mortality rates in the middle age years [3] as well as environmental influences in early life such as poor intrauterine growth [43] leading to earlier and more progressive disease.

There were limitations to this study. Indigenous participants were recruited from communities who in turn were selected based on the likelihood that their health service had the capacity and the organizational support to deliver the intervention and so results may not be generalizable to all Aboriginal and Torres Strait Islander communities. Although interesting, our results related to health literacy should be treated with caution. Current assessment tools, including the TOFHLA survey, essentially measure reading skills and may not differentiate between reading ability and background knowledge of scientific health concepts and/or cultural differences in the understanding of health and health care [44]. Further, given that only people with diabetes and HbA1c levels $\geq 8.5 \%$ were included, the results may not be generalizable to all Indigenous Australians with diabetes. For some clinical variables there were missing data although the characteristics of participants with missing data were similar to those with data recorded. This pragmatic trial in a highly mobile population reflects the realities of delivering optimal diabetes care. Prevalence of albuminuria may be overestimated as urinary ACR calculations were based on a single sample and microscopy, culture and sensitivity was not performed at the time of collection.

Effective and sustained delivery of diabetes care by Indigenous Health Workers who are linguistically and culturally close to their clients has previously been demonstrated in the Torres Strait $[12,16,45]$. This resulted in much improved continuity of care compared to that provided by an itinerant and often inexperienced non-Indigenous workforce. It is our view that better utilisation of the Indigenous health workforce has the potential to increase the health standards in high need populations through the delivery of more culturally safe health care and greater engagement with the extended family of people living with diabetes. 


\section{Conclusion}

As such, these results demonstrate a high potential for improved culturally-sound integrated community-based management of diabetes and other comorbid conditions in this very high risk population. They also provide further evidence for including albuminuria in cardiovascular risk calculation in clinical settings in addition to traditional risk factors [46].

\section{Competing interests}

The authors declare they have no competing interests.

\section{Authors' contributions}

RM developed the research design and project proposal, undertook community consultation and obtained grant funding and reviewed the paper. ST and CP led participant recruitment and data collection. BS managed the study. DJ, RM and PC undertook the intellectual planning for the data analysis. DJ undertook the data analysis and wrote the first draft of the paper. RM, PC and $\mathrm{KD}$ assisted with data analysis, data interpretation and reviewing drafts of the paper. All authors read and approved the final manuscript.

\section{Acknowledgements}

The authors acknowledge Adrian Esterman from the University of South Australia for providing statistical assistance during analysis of the results The GBACC study is supported by the National Health and Medical Research Council (NHMRC project grant number 570149). RM is supported by a NHMRC Practitioner Fellowship.

\section{Author details}

${ }^{1}$ Aboriginal Health Council of South Australia, 9 King William Road, Adelaide, SA 5061, Australia. ${ }^{2}$ College of Public Health, Medical and Veterinary Sciences, James Cook University, Cairns, Australia. ${ }^{3}$ School of Pharmacy and Medical Sciences, University of South Australia, Baker IDI Heart and Diabetes Institute, Adelaide, Australia. ${ }^{4}$ School of Population Health, University of South Australia, Adelaide, Australia.

\section{Received: 11 May 2014 Accepted: 19 March 2015}

Published online: 03 April 2015

\section{References}

1. Australian Bureau of Statistics. National Aboriginal and Torres Strait Islander Health Survey: Australia, 2004-05. Canberra: ABS; 2006.

2. Australian Institute of Health and Welfare. Aboriginal and Torres Strait Islander health performance framework 2010: detailed analyses. Canberra: AlHW; 2011.

3. Minges KE, Zimmet P, Magliano DJ, Dunstan DW, Brown A, Shaw JE. Diabetes prevalence and determinants in Indigenous Australian populations: A systematic review. Diabetes Res Clin Pract. 2011;93(2):139-49.

4. Vos T, Barker B, Begg S, Stanley L, Lopez AD. Burden of disease and injury in Aboriginal and Torres Strait Islander Peoples: The indigenous health gap. Int J Epidemiol. 2009;38(2):470-7.

5. Donald M, Naylor M, Parekh S, Orlandi A. Hospital Separations, Avoidable Admissions, Health Service Utilisation \& Quality of Care. Baseline evaluation of the Queensland Strategy for Chronic Disease 2005-2015 (No. 2). St Lucia: University of Queensland, Australia; 2007.

6. Li SQ, Gray NJ, Guthridge SL, Pircher SLM. Avoidable hospitalisation in Aboriginal and non-Aboriginal people in the Northern Territory. Med J Aust. 2009;190(10):532-6.

7. Ansari Z, Laditka JN, Laditka SB. Access to health care and hospitalization for ambulatory care sensitive conditions. Med Care Res Rev. 2006;63(6):719-41.

8. Wagner EH, Austin BT, Davis C, Hindmarsh M, Schaefer J, Bonomi A. Improving chronic illness care: Translating evidence into action. Health Aff (Millwood). 2001;20(6):64-78.

9. Queensland Government. Queensland Strategy for Chronic Disease 2005-2015. Brisbane: Queensland Health; 2005.

10. Northern Territory Chronic Conditions Prevention and Management Strategy 2010-2020. Darwin: Department of Health and Families; 2009.
11. Si D, Bailie R, Dowden M, Kennedy C, Cox R, O'Donoghue L, et al. Assessing quality of diabetes care and its variation in Aboriginal community health centres in Australia. Diabetes Metab Res Rev. 2010;26(6):464-73.

12. McDermott $R$, Tulip F, Schmidt B, Sinha A. Sustaining better diabetes care in remote indigenous Australian communities. Br Med J. 2003;327(7412):428-30.

13. McDermott RA, McCulloch BG, Campbell SK, Young DM. Diabetes in the Torres Strait Islands of Australia: Better clinical systems but significant increase in weight and other risk conditions among adults, 1999-2005. Med J Aust. 2007;186(10):505-8.

14. Phillips LS, Branch Jr WT, Cook CB, Doyle JP, El-Kebbi IM, Gallina DL, et al. Clinical inertia. Ann Intern Med. 2001;135(9):825-34.

15. Bailie RS, Si D, Robinson GW, Togni SJ, d'Abbs PHN. A multifaceted healthservice intervention in remote aboriginal communities: 3-year follow-up of the impact on diabetes care. Med J Aust. 2004;181(4):195-200.

16. McDermott RA, Schmidt BA, Sinha A, Mills P. Improving diabetes care in the primary healthcare setting: $A$ randomised cluster trial in remote indigenous communities. Med J Aust. 2001;174(10):497-502.

17. Hoy WE, Kondalsamy-Chennakesavan SN, Nicol JL. Clinical outcomes associated with changes in a chronic disease treatment program in an Australian aboriginal community. Med J Aust. 2005;183(6):305-9.

18. Si D, Bailie RS, Togni SJ, D'Abbs PHN, Robinson GW. Aboriginal health workers and diabetes care in remote community health centres: A mixed method analysis. Med J Aust. 2006;185(1):40-5.

19. Schmidt B, Wenitong M, Esterman A, Hoy W, Segal L, Taylor S, et al. Getting better at chronic care in remote communities: study protocol for a pragmatic cluster randomised controlled of community based management. BMC Public Health. 2012;12(1):1017.

20. Parker RM, Baker DW, Williams MV, Nurss JR. The test of functional health literacy in adults: A new instrument for measuring patients' literacy skills. J Gen Intern Med. 1995;10(10):537-41.

21. Johnson DW, Jones GRD, Mathew TH, Ludlow MJ, Doogue MP, Jose MD, et al. Chronic kidney disease and automatic reporting of estimated glomerular filtration rate: New developments and revised recommendations. Med J Aust. 2012;197(4):222-3.

22. Johnson DW, Atai E, Chan M, Phoon RKS, Scott C, Toussaint ND, et al. KHACARI guideline: Early chronic kidney disease: Detection, prevention and management. Nephrology. 2013;18(5):340-50.

23. Hoy WE, Mathews JD, McCredie DA, Pugsley DJ, Hayhurst BG, Rees M, et al. The multidimensional nature of renal disease: Rates and associations of albuminuria in an Australian Aboriginal Community. Kidney Int. 1998;54(4):1296-304

24. Rowley KG, Iser DM, Best JD, O'Dea K, Leonard D, McDermott R. Albuminuria in Australian Aboriginal people: Prevalence and associations with components of the metabolic syndrome. Diabetologia. 2000:43(11):1397-403.

25. Thomas M, Weekes AJ, Thomas MC. The management of diabetes in indigenous Australians from primary care. BMC Public Health. 2007;7:303

26. Maple-Brown $\sqcup$, Cunningham J, Hodge AM, Weeramanthri T, Dunbar T, Lawton PD, et al. High rates of albuminuria but not of low eGFR in Urban Indigenous Australians: The DRUID Study. BMC Public Health. 2011;11:346.

27. Hoy WE, Kincaid-Smith $P$, Hughson MD, Fogo AB, Sinniah R, Dowling J, et al. CKD in aboriginal Australians. Am J Kidney Dis. 2010;56(5):983-93.

28. McDermott RA, Tulip F, Schmidt B. Diabetes care in remote northern Australian indigenous communities. Med J Aust. 2004;180(10):512-6.

29. Davis TME, Hunt K, McAullay D, Chubb SAP, Sillars BA, Bruce DG, et al. Continuing disparities in cardiovascular risk factors and complications between aboriginal and Anglo-Celt Australians with type 2 diabetes: The fremantle diabetes study. Diabetes Care. 2012;35(10):2005-11.

30. McDonald SP, Maguire GP, Hoy WE. Renal function and cardiovascular risk markers in a remote Australian Aboriginal community. Nephrol Dial Transplant. 2003;18(8):1555-61.

31. Maple-Brown $\sqcup$, Hughes JT, Lawton PD, Jones GRD, Ellis AG, Drabsch K, et al. Accurate assessment of kidney function in indigenous australians: The estimated GFR study. Am J Kidney Dis. 2012;60(4):680-2.

32. Basi S, Fesler P, Mimran A, Lewis JB. Microalbuminuria in type 2 diabetes and hypertension: a marker, treatment target, or innocent bystander? Diabetes Care. 2008;31 Suppl 2:S194-201.

33. Chronic Kidney Disease Prognosis Consortium. Association of estimated glomerular filtration rate and albuminuria with all-cause and cardiovascular mortality in general population cohorts: a collaborative meta-analysis. Lancet. 2010;375(9731):2073-81. 
34. Viberti G, Wheeldon NM. Microalbuminuria reduction with valsartan in patients with type 2 diabetes mellitus: A blood pressure-independent effect. Circulation. 2002;106(6):672-8.

35. Grant RW, Cagliero E, Dubey AK, Gildesgame C, Chueh HC, Barry MJ, et al. Clinical inertia in the management of Type 2 diabetes metabolic risk factors. Diabet Med. 2004;21(2):150-5.

36. Schillinger D, Grumbach K, Piette J, Wang F, Osmond D, Daher C, et al. Association of health literacy with diabetes outcomes. J Am Med Assoc. 2002;288(4):475-82.

37. Berkman ND, Sheridan SL, Donahue KE, Halpern DJ, Crotty K. Low health literacy and health outcomes: An updated systematic review. Ann Intern Med. 2011;155(2):97-107.

38. Dunstan DW, Zimmet PZ, Welborn TA, De Courten MP, Cameron AJ, Sicree RA, et al. The rising prevalence of diabetes and impaired glucose tolerance: The Australian diabetes, obesity and lifestyle study. Diabetes Care. 2002;25(5):829-34.

39. McDermott R, Rowley KG, Lee AJ, Knight S, O'Dea K. Increase in prevalence of obesity and diabetes and decrease in plasma cholesterol in a central Australian Aboriginal community. Med J Aust. 2000;172(10):480-4.

40. National Association of Diabetes Centres. Final Report. ANDIAB. Australian National Diabetes Information Audit \& Benchmarking. Canberra: NADC; 2011.

41. O'Dea K, Rowley KG. Macrovascular disease risk factors and insulin resistance in Aboriginal and Torres Strait Islander people. J Diabetes Complications. 2002;16(1):9-16

42. Swinburn BA, Ley SJ, Carmichael HE, Plank LD. Body size and composition in Polynesians. Int J Obes. 1999;23(11):1178-83.

43. Godfrey KM, Lillycrop KA, Burdge GC, Gluckman PD, Hanson MA. Epigenetic mechanisms and the mismatch concept of the developmental origins of health and disease. Pediatr Res. 2007;61 (5 PART 2 SUPPL):5R-10.

44. Nielsen-Bohlman L, Panzer AM, Kindig DA. Health Literacy: A Prescription to End Confusion. Washington D.C: National Academies Press; 2004.

45. McDermott R, Segal L. Cost impact of improved primary level diabetes care in remote Australian indigenous communities. Australian J Primary Health. 2006;12(2):124-30

46. McDermott RA, McCulloch B, Li M. Glycaemia and Albuminuria as predictors of coronary heart disease in aboriginal and torres strait islander adults: A north Queensland cohort. Med J Aust. 2011;194(10):514-8.

\section{Submit your next manuscript to BioMed Central and take full advantage of:}

- Convenient online submission

- Thorough peer review

- No space constraints or color figure charges

- Immediate publication on acceptance

- Inclusion in PubMed, CAS, Scopus and Google Scholar

- Research which is freely available for redistribution 